\title{
INFORMATION FUSION FOR MAKING CREDIT DECISION OF PPP ROAD PROJECTS
}

\author{
Boeing Singh Laishram \\ $\mathrm{Ph}$ D Research Scholar \\ BTCM Division \\ Department of Civil Engineering \\ Indian Institute of Technology Madras \\ Chennai-600 036, India \\ boeing@iitm.ac.in
}

\author{
Satyanarayana N. Kalidindi \\ Professor \\ BTCM Division \\ Department of Civil Engineering \\ Indian Institute of Technology Madras \\ Chennai - 600 036, India \\ satyakn@iitm.ac.in
}

\begin{abstract}
The systematic aggregation of information is crucial in decision making. If the information is derived from various sources, then the information fusion problem can be transformed into a multi-criteria decision making model. The information fusion problem, thus, is reduced to (a) determining the structure of the hierarchy to be used; (b) computing the degree of satisfaction of criteria based on the observed features; and (c) the nature of the connectives at each node of the hierarchy. The degrees of satisfaction of criteria need to be determined in linguistic terms in case the information is not precise and complete in nature. In view of this, the suitable aggregation operators are the fuzzy connectives. Choquet fuzzy integral is one of the preferred fuzzy connectives for aggregating the information in case there are interactions between the criteria. This paper discusses the application of Choquet fuzzy integral in information fusion with the help of the information the lenders used in making credit decision to Public Private Partnership (PPP) road projects. Lenders to PPP road projects are more concerned with the debt servicing capability of the projects. Lenders aggregate information on the performance of the project with respect to the various aspects, which can influence the debt servicing capability, of the project to assess whether the risk/reward profile of the project match their risk/reward appetite. Information fusion using Choquet fuzzy integral facilitates systematic aggregation of the information and enables the lenders to derive the degree of match between their risk/reward appetite and the risk/reward profile of the project.
\end{abstract}

\section{KEYWORDS}

Public Private Partnerships, Project Finance, Debt Financing, and Information Fusion

\section{INTRODUCTION}

The importance of systematically aggregating the information for decision making in the construction management perspective requires no further emphasis. A typical decision making problem like making a decision to select a construction firm as the EPC contractor for a project requires aggregation of the information on the various dimensions such as experience, financial strength, managerial and engineering capabilities. The information on these dimensions is derived from diverse sources. Krishnapuram and Lee had suggested that information from various sources can be aggregated in a hierarchical manner with multilevel framework [1]. The node in the hierarchical network can be modelled as criterion and the information aggregation problem can be converted to a multi-criteria decision making model, with the input to the node as degrees of satisfaction of the sub-criteria and the output as the aggregated degree of satisfaction of 
the criterion. The information fusion problem can, thus, be reduced to: (a) determining the structure of the hierarchy to be used; (b) computing the degree of satisfaction of criteria based on the observed features; and (c) the nature of the connectives at each node of the hierarchy.

The degrees of satisfaction of the criteria can be determined objectively if the information is precise and accurate in nature. However, if the information is not precise and complete in nature, the degrees of satisfaction of the criteria can best be computed in the form of linguistic terms. Grabisch had classified the appropriate operators available for aggregating linguistic terms into tnorm, t-conorm, and averaging operators [2]. These operators are suitable for aggregating the information when the criteria are linearly independent. When the criteria are not mutually independent, these operators fail to yield accurate results on the aggregated information. Fuzzy integral is reported to be the preferred operator for aggregating the information in case there are interactions between the criteria [3].

Highway projects procured through Public Private Partnership (PPP) route are financed with funding from various funding agencies. Commercial banks have been one of the funding agencies providing major portion of the debt financing to PPP road projects. When debt financing is provided on a Project Finance basis, lenders have limited or no access to the sponsors' assets. Lenders look at the revenue earning potential of the project as the major source for servicing the debt. They are also more concerned with the downside risks associated with the projects that can have adverse effects on the project capacity to service debt [4]. Lenders prior to underwriting the loan undertake a loan approval process wherein the lender's project finance team examines the various aspects of the project, namely technical, commercial, financial, marketing, management factors, sponsor's financial strength and experience, security arrangements, and credit support. The information on these aspects are derived from various sources, including project feasibility study reports, concession agreement, EPC and O\&M contracts, shareholders agreement, financial model, and information memorandum [5]. The lenders aggregate information on these aspects to examine whether the risk/reward profile of the project under examination matches their risk/reward appetite. The degree of match influences the lender's decision to advance project finance loan to the project. This paper discusses the application of fuzzy integral in aggregating information for decision making using the information on the various aspects of PPP road projects that the lenders take into account in making the credit decision.

\section{FUZZY MEASURE AND FUZZY INTEGRAL}

Fuzzy integral is the general term for integral with respect to the fuzzy measure [6]. The concept of fuzzy integral was first put forward by Sugeno, in the early seventies, who considered fuzzy measures and suggested fuzzy integral as a generalization of Lebesgue integrals.

\subsection{Fuzzy Measure}

Fuzzy measure is a generalization of the notion of measure in mathematical analysis and it relaxes the condition of additivity for the classical measure and only assume monotonocity [7]. There are several classes of fuzzy measures but Sugeno measure is the most commonly used fuzzy measure in human subjective evaluation, pattern recognition, and decision analysis. Computing the Sugeno fuzzy measure is made simpler with the help of the $\lambda$ rule. The properties of Sugeno fuzzy measure are illustrated below $[8,9]$.

Let $g$ be a fuzzy measure on a finite set $X=\left\{x_{1}\right.$, $\left.x_{2}, x_{3}, \ldots \ldots x_{n}\right\}$. The function $g$ such that $g\left(x_{i}\right): x_{i}$ $\in X \rightarrow[0,1]$ is known as the fuzzy density function. For simplicity, $g\left(x_{i}\right)$ is denoted as $g^{i}$. The fuzzy measure, $g_{\lambda}(A)$, of a finite set, say $A=$ $\left\{x_{1}, x_{2}\right\} \subset X$, can be obtained from the fuzzy densities as follows:

$g_{\lambda}\left(\left\{x_{1}, x_{2}\right\}\right)=g^{1}+g^{2}+\lambda g^{1} g^{2}$

and, generally 


$$
\begin{gathered}
g_{\lambda}\left(\left\{x_{i_{1}} \cdots x_{i_{m}}\right\}\right)=\sum_{j=1}^{m} g^{i_{j}}+\lambda \sum_{j=1}^{m-1} \sum_{k=j+1}^{m} g^{i_{k}}+\cdots \\
\cdots+\lambda^{m-1} g^{i_{1}} \cdots g^{i_{m}} \\
=\left[\prod_{x_{i} \in A}\left(1+\lambda g^{i}\right)-1\right] / \lambda, \quad \lambda \notin 0
\end{gathered}
$$

We know that the fuzzy measure of the finite set $X=\left\{x_{1}, x_{2}, \ldots . x_{n}\right\}$ is equal to one and, then, Equation 2 reduces to Equation 3.

$$
\lambda+1=\prod_{i=1}^{n}\left(1+\lambda g^{i}\right)
$$

If we denote the right hand side as $G(\lambda)$, then the solutions to the above equation are the intersections between the polynomial $G(\lambda)$ and $\lambda+$ 1.

If all the fuzzy densities $g^{i}, i=1,2,3 \ldots . n$, are known then, using the above equation, fuzzy measure of any set $A$, where $A \subset X$, can be computed. For the information fusion problem, we interpret the fuzzy density value $g^{i}$ as the grade of importance of some source $x_{i}$, towards the final evaluation. The $g_{\lambda}$ fuzzy measure of an arbitrary set $\boldsymbol{A}$ represents the importance grade of the set of sources denoted by $\boldsymbol{A}$ towards final decision.

\subsection{Fuzzy Integral}

Different classes of fuzzy integral had been introduced since the introduction of the concept of Sugeno fuzzy integral by Sugeno in the seventies. Murofushi and Sugeno proposed an integral, Choquet integral, which is coherent with the Lebesque integral. Choquet integral is the extension of the ordinary integral and the most natural fuzzy integral [6]. The concept of Choquet fuzzy integral is described below [10].

Let $h\left(x_{1}\right), h\left(x_{2}\right), h\left(x_{3}\right) \ldots . . h\left(x_{n}\right)$ be the objective evidences provided by the criteria $x_{1}, x_{2}$, $x_{3} \ldots \ldots x_{n}$, respectively, and $g$ is the $\lambda$ fuzzy measure, then we can construct Choquet fuzzy integral as
(C) $\int h(.) \circ g($.

The above Choquet fuzzy integral reduces to the following form when $X$ is finite:

$$
\begin{aligned}
& E_{g}(h)=\sum_{i=1}^{n}\left[h\left(x_{i}\right)-h\left(x_{i-1}\right)\right] g\left(A_{i}\right) \\
& \text { Where, } \\
& h\left(x_{1}\right) \leq h\left(x_{2}\right) \leq \cdots \leq h\left(x_{n}\right), \\
& h\left(x_{0}\right)=0, \text { and } \\
& g\left(A_{i}\right)=g\left(x_{i}, x_{i+1}, x_{i+2} \ldots . ., x_{n}\right)
\end{aligned}
$$

For computing the Choquet fuzzy integral, the required inputs are the fuzzy densities $(g)$ and objective evidences $(h)$.

\section{APPLICATION}

The Bank for International Settlements studied the aspects considered by the banks in qualifying the project finance loans [11]. Figure 1 shows the aspects with their elements. The application of fuzzy integral in determining the extent to which the risk/reward profile of the project matches the risk/reward appetite of the lenders is explained using the aspects and elements of Figure 1. Prior to aggregation of information using fuzzy integral, three sets of inputs relating to the elements in the level 3 of the Figure 1 are required to be obtained. The inputs are: (i) degrees of satisfaction of the criteria; (ii) grades of importance of the criteria; and (iii) four rating levels, expressed in terms of triangular fuzzy numbers, over the possible values of the elements representing the different levels of lenders' risk/reward appetite.

\subsection{Determination of the Grade of Importance}

The grades of importance of the elements are elicited in terms of linguistic terms "Unimportant (UI)", "Slightly Important (SI)", "Fairly Important (FI)", "Important (I)", and "Very Important (VI)". These linguistics terms are transformed into Triangular Fuzzy Numbers to facilitate fuzzy operations. The equivalent fuzzy numbers for these linguistics terms "UI", "SI", "FI", "I", and "VI" are respectively $(0.0,0.0,0.3),(0.0,0.25$, $0.5),(0.3,0.5,0.7),(0.5,0.75,1)$, and $(0.7,1,1)$. 


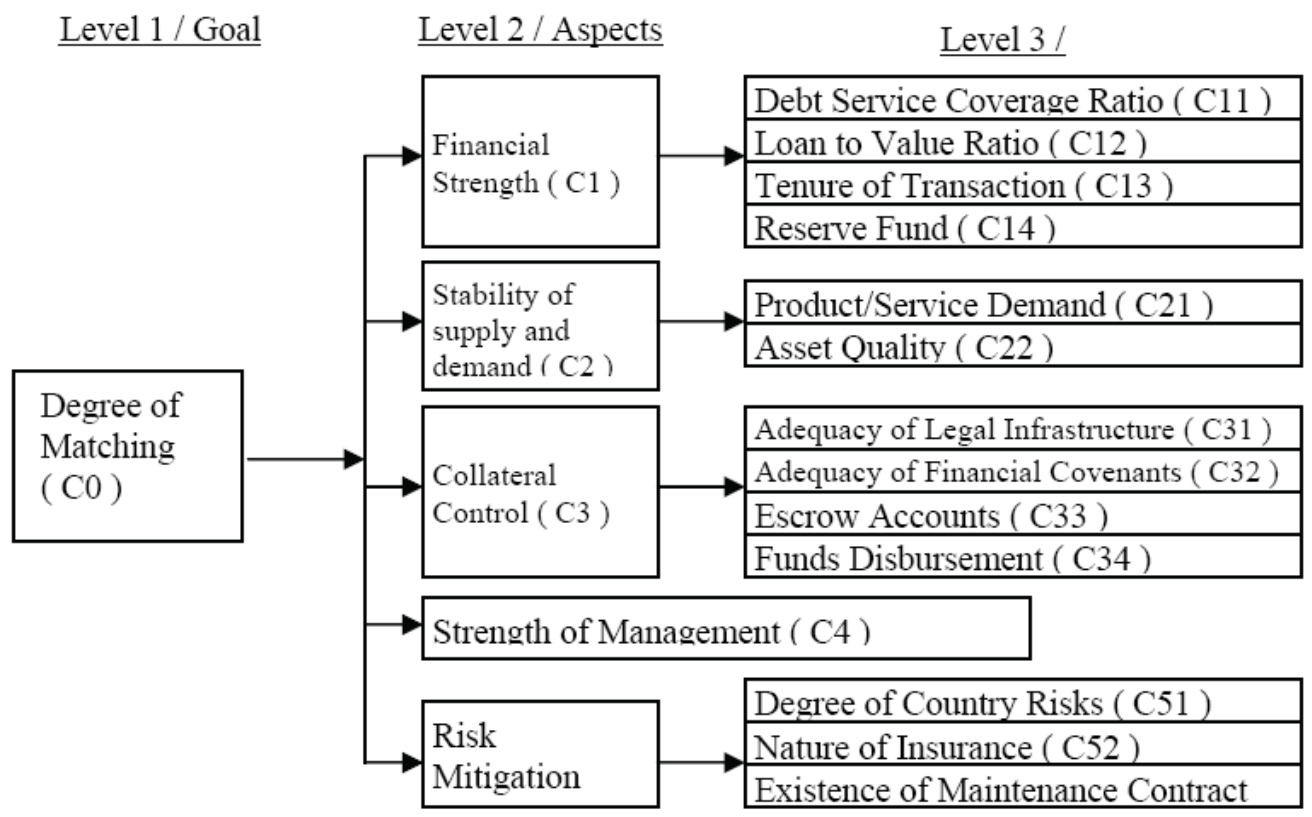

Figure 1 Aspects and Elements Used by Banks for Qualifying Project Finance Loan

The grades of importance of the project under consideration with respect to the elements are obtained in terms of the above linguistic terms. The corresponding triangular fuzzy numbers are then defuzzified using discretisation method to yield the fuzzy densities and enable computation are used to compute the value of $\lambda$. For example, the basic hierarchical structure under the parent node $\mathrm{C} 1$, the grades of importance of the child nodes under $\mathrm{C} 1$ are used to compute the value of $\lambda$ for that basic hierarchical structure using Equation 3. The values of $\lambda$ 's for the other basic hierarchical structures under the parent nodes $\mathrm{C} 2$, $\mathrm{C} 3$, and $\mathrm{C} 5$ are also computed in the similar fashion.

\subsection{Determination of the Objective Evidence}

Prior to determination of the objective evidences, it is required to express the possible values the elements can attain in terms of four different fuzzy rating levels, namely "Very Undesirable (VU)", "Undesirable (U)", "Desirable (D)", and "Very Desirable (VD)". The elements like Debt Service Coverage Ratio (DSCR) and Loan to Value (LTV) ratio take numerical values. These values are transformed into four different triangular fuzzy numbers representing the four fuzzy rating levels. Figure 2 exemplifies the four fuzzy rating levels of numerical ratios such as DSCR. The elements, except DSCR, LTV, and Tenure of Transaction, are measured using experts' judgments. With respect to these elements, the degrees of achievement are measured in terms of triangular fuzzy numbers in the interval $[0,100] .100$ points signify the most satisfactory achievement while 0 denotes the least. Figure 3 shows the possible membership functions of one of the elements requiring subjective assessment in measuring the degree of achievement such as "Adequacy of Legal Infrastructure".

For the project under consideration, experts are asked to rate how the project performs with respect to the elements in terms of triangular fuzzy numbers $(a, b, d)$. The values of $a, b$, and $c$ refers to the least possible, most likely, and most possible values. 


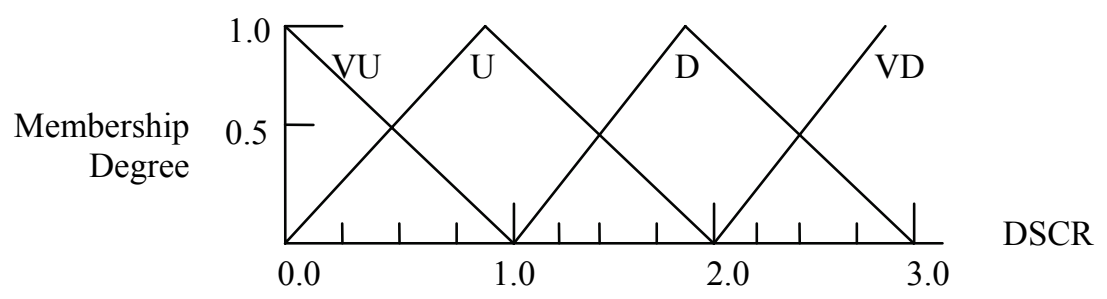

Figure 2 Membership Functions of the Four Rating Levels of DSCR

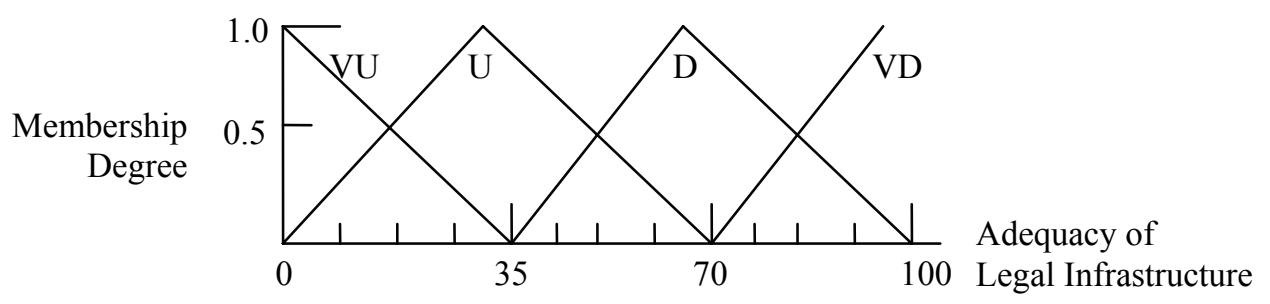

Figure 3 Membership Functions of the Four Rating Levels of "Adequacy of Legal Infrastructure"

These triangular fuzzy numbers give the degrees of satisfaction of criteria. These triangular fuzzy numbers are mapped onto the corresponding membership functions of the concerned elements. The degrees of similarity of the triangular fuzzy number, representing the degree of satisfaction of the criterion, to the four fuzzy rating levels of the corresponding element give the objective evidences of the concerned element.

\subsection{Aggregation Procedure}

Aggregation of the objective evidences is done in a bottom-up approach for each rating level. For the basic hierarchical structure under the parent criterion $\mathrm{C}$, the values of the objective evidences of $\mathrm{C} 11, \mathrm{C} 12, \mathrm{C} 13$, and $\mathrm{C} 14$ relative to the fuzzy rating level "Very Undesirable" are arranged in ascending order. Fuzzy measures in accordance with the different combinations of fuzzy densities of the elements as per Equation 5 are computed using Equation 3. The objective evidence of the parent node $\mathrm{C} 1$ with respect to the lowest fuzzy rating "VU" is computed using the objective evidences and fuzzy measures of the child nodes with the help of the Equation 5. The objective evidences with respect to the lowest rating level are computed in similar fashion for the parent nodes $\mathrm{C} 2, \mathrm{C} 3$, and $\mathrm{C} 5$. Using the objective evidences of $\mathrm{C} 1, \mathrm{C} 2, \mathrm{C} 3, \mathrm{C} 4$, and $\mathrm{C} 5$ and the corresponding fuzzy measures objective evidence with respect to the lowest rating level is computed for the node at the level 1 . The same aggregation procedure is adopted for the entire hierarchical structure for the remaining fuzzy rating levels. The objective evidences of the node at level 1 derived from the aggregation process give the measure on the degrees of matching with respect to the four rating levels. Matching to a significant extent to the rating levels " $D$ " or "VD" signifies a match between the risk/reward profile of the project and risk/reward appetite of the lenders.

\section{CONCLUSIONS}

Lenders before making credit decision aggregate information on the aspects of the PPP projects to determine whether the risk/reward profile of the project matches their risk/reward appetite. The nature of the information available at the time of decision making is not precise and accurate. The review of literature on information fusion has indicated Choquet fuzzy integral as one of the aggregation operators for fusion of imprecise and inaccurate information. This paper discusses the 
concepts of Choquet fuzzy integral and fuzzy measure. The paper also elaborates the application of Choquet fuzzy integral to assess the degree of match between the risk/reward profile of the project and risk/reward appetite of the lenders with the help of the aspects used by the banks in qualifying project finance loans. The aggregated information on the degree of match can facilitate lenders to make decision on advancing project finance loan to PPP projects.

\section{REFERENCES}

[1] Krishnapuram, R. and J. Lee, Fuzzyconnective-based hierarchical aggregation networks for decision making. Fuzzy Sets and Systems, 1992. 46(1): p. 11-27.

[2] Grabisch, M., Fuzzy integral in multicriteria decision making. Fuzzy Sets and Systems, 1995. 69(3): p. 279-298.

[3] Grabisch, M., The application of fuzzy integrals in multicriteria decision making. European Journal of Operational Research, 1996. 89(3): p. 445-456.

[4] Walker, C. and A.J. Smith, Privatized infrastructure: the Build Operate Transfer approach. 1995, London: Thomas Telford.

[5] Yescombe, E.R., Principles of Project Finance. 2002, California: Academic Press.
[6] Grabisch, M., T. Murofushi, and M. Sugeno, Fuzzy measures and integrals: Thoery and applications. First ed. Studies in fuzziness and soft computing, ed. J. Kacprzyk. Vol. 40. 2000, New York: Physica-Verlag. 477.

[7] Ying, M., Linguistic quantifiers modeled by Sugeno integrals. Artificial Intelligence, 2006. 170(6-7): p. 581-606.

[8] Tahani, H. and J.M. Keller, Information fusion in computer vision using fuzzy integral. IEEE Transactions on Systems, Man and Cybernetics, 1990. 20(3): p. 733-741.

[9] Leszczynski, K., P. Penczek, and W. Grochulski, Sugeno's fuzzy measure and fuzzy clustering. Fuzzy Sets and Systems, 1985. 15(2): p. 147-158.

[10] Chiang, J.-H., Choquet fuzzy integralbased hierarchical networks for decision analysis. IEEE Transactions on Fuzzy Systems, 1999. 7(1): p. 63-71.

[11] BIS, Working paper on the internal ratings-based approach to specialised lending exposures. 2001, Basel Committee on Banking Supervision, Bank for International Settlements. 